VOL. $26(1982), 249-262$.

\title{
NONLINEAR DIFFUSION IN A FINITE LAYER*
}

\author{
J.-Y. Parlange, D.A. Lockington and R.D. Braddock \\ Communicated by James M. Hill
}

\begin{abstract}
As a liquid enters a porous medium the transition from dry to wet occurs in a narrow, unsaturated region at the wetting front, where the liquid content increases very rapidly. When this unsaturated zone approaches an impermeable wall, further rapid changes of the water content take place and liquid intake ceases quickly. We are interested in finding the liquid intake as a function of time when liquid movement obeys a diffusion equation. The rapid changes occurring in the phenomenom make the problem strongly nonlinear and for realistic diffusivities numerical solutions can be expensive. However, we present here an optimization principle which yields an analytical approximation of the liquid intake. The result is more accurate when nonlinear effects are more pronounced. Comparison with numerical results shows that optimization is remarkably accurate.
\end{abstract}

\section{Introduction}

Problems of liquid movement in porous media have been studied "in several separate fields of natural science and in a large number of branches of technology" [18]. A porous medium is formed by interconnected pore spaces forming channels available for liquid and, more generally, any

Received 20 March 1982.

* This paper is based on an invited lecture given at the Australian Mathematical Society Applied Mathematics Conference held in Bundanoon, February 7-11, 1982. Other papers delivered at this Conference appear in Volumes 25 and 26 . 
fluid movement. A typical and most important porous medium is a soil and the movement of water and oil in a soil has obvious practical applications.

Under the simplest circumstances, neglecting possible swelling of the medium, assuming no effect due to temperature and solute gradients, neglecting gravity and the resistance of the displaced fluid (usually air) as well as other secondary effects, the movement of the liquid can be described by the diffusion equation expressing mass conservation, [18], $[10]$,

$$
\frac{\partial \Theta}{\partial t}=\partial \frac{[D \partial \Theta / \partial x]}{\partial x}
$$

Here $\theta$ is the liquid content which we shall express on a scale of 0 , dry, to 1 , saturated; $x$ is the distance from the surface where the liquid enters the medium and, $t$ is the time. The diffusivity, $D$, entering (1) has a strong dependence on $\theta$. For instance, [19], $D$ can often be described by an exponential,

$$
D=D_{0} \exp n \theta \text {, }
$$

where $n$ is the order $8,[19]$. To solve the problem initial and boundary conditions are needed, for instance,

$$
\begin{gathered}
t \leq 0, \theta=0, \\
x=0, t>0, \quad \theta=1 .
\end{gathered}
$$

If the medium extends to $x \rightarrow \infty$, then

$$
x \rightarrow \infty, \quad \theta=0 \text {. }
$$

When (3), (4) and (5) hold, no length scale enters the problem and a similarity variable, $\phi$, exists with

$$
\phi=x t^{-\frac{1}{2}}
$$

with (1) reducing to

$$
\phi=-2 d \frac{[D d \theta / d \phi]}{d \theta}
$$

This equation has been used in the past to measure $D,[5],[7]$. Although we limit ourselves to one spatial dimension, $x$, there is no difficulty in finding similarity solution in cylindrical and spherical problems, [17], [15], [16], [12]. When $D$ has the exponential form (2) or obeys a power 
law, then (7) has a first integral which simplifies greatly the numerical integration of (7), [13], [15], [16], [4]. In general, when $D$ is arbitrary, an optimization principle exists which yields the flux at $x=0,[8]$, [1]. This principle has also been found quite useful to solve (7) numerically [2] or to obtain analytical approximations to the solution, [11].

Condition (5) is at best an approximation since porous media are often far from infinite, for example, a soil usually has a layered structure. If we call $L$ the thickness of the layer or slab of porous material, then condition (5) must be replaced by a condition at $x=L$. For an impermeable surface at $x=L,(5)$ is replaced by

$$
\frac{\partial \Theta}{\partial x}=0 \text { at } x=L \text {. }
$$

Introduction of a length scale, $L$, means that the similarity solution does not hold any more. More specifically during the initial stages liquid intake (5) will hold approximately, but as soon as the wetting front interacts with the impermeable surface, the liquid intake will be less than would be predicted using (5).

\section{Optimization}

Let us call $t_{T}$ the transition time during which the wetting front has yet to interact significantly with the impermeable wall. Hence (5) and (7) hold for $t<t_{T}$ and previous optimization results can be used, [8], [10]. These are now summarized.

As long as $D$ increases rapidly we define a small parameter, $\varepsilon$, by

$$
\varepsilon=\int_{0}^{1}(1-\theta) D d \theta / \int_{0}^{1} D d \theta .
$$

For instance, if (2) holds, $\varepsilon=O\left(n^{-1}\right)$; as $\varepsilon \rightarrow 0$, the behaviour of $D$ is that of a delta-function and terms of order $\varepsilon$ represent the deviation from a delta-function. In the limit of $\varepsilon \rightarrow 0$ the lefthand sides of (1) and (7) become negligible, or, for $t<t_{T}$, we can write

$$
\phi=\lambda \int_{\theta}^{1} f D d \bar{\theta}
$$


where $\lambda$ is some unknown constant and $f$ is a function of $\theta$, with $f(1)=1$. If the left hand side of (7) was truly negligible, we would have $f=1$ for all $\theta^{\prime} s$. In general $f$ is a mildly varying function of $\theta$, as compared with the variation of $D$. For an arbitrary choice of $f$, for example, $1, \theta, \theta^{\frac{1}{2}},[8],[6], \phi$ in (10) has in general an error of order $\varepsilon$. The purpose of optimization is to determine the liquid intake, represented by the sorptivity $S$, with

$$
S=\int_{0}^{1} \phi d \theta
$$

which has only an error of order $\varepsilon^{2}$ even though $\phi$ has an error $O(\varepsilon)$. This is insured by choosing $\lambda$ for any $f$ such that

$$
\int_{0}^{1} \phi^{2} d \theta=2 \int_{0}^{1} D d \theta
$$

Note that (12) results from integrating (7) twice. Up to error of order $\varepsilon^{2}, S$ can be given by, [8], [6],

$$
S^{2}=\int_{0}^{1}(1+\theta) D d \theta \text {, or } 2 \int_{0}^{1} \theta^{\frac{1}{2}} D d \theta \text {. }
$$

Both results are extremely accurate, [6]. Further iterations can improve $S$ and reduce the error to $O\left(\varepsilon^{3}\right),[14]$. In practice such refinement will rarely be necessary. To extend the previous result for $t>t_{T}$, the value of $t_{T}$ must first be estimated as the time when the wetting front initially comes into contact with the impermeable wall. The position of the wetting front, like $S$, is determined with an error $O\left(\varepsilon^{2}\right)$, whatever $f$ is in (10) as long as (12) or (13) holds, [9]. Or, writing that $x=L$ at $t=t_{T},(10)$ and (13) yield at once

$$
\left.t_{T} / L^{2}=\left(\int_{0}^{1} \theta D d \theta\right)^{2} /\left[\iint_{0 .}^{1} D d \theta\right)^{2} \int_{0}^{1} D(1+\theta) d \theta\right] .
$$

We may note that as $\Theta \rightarrow 0$, usually $D \neq 0$, as shown by (2) for instance. Then some diffusion takes place ahead of the wetting front, [2]. Although 
the amount of material involved is exponentially small it implies that some interaction with the impermeable wall takes place before $t=t_{T}$.

For $t>t_{T}$, we now write, following the same reasoning leading to (10),

$$
x=h \int_{\theta}^{1} f D d \theta \text {, }
$$

with $f(1)=1$, but now $h$ is a function of $t$, since the similarity solution does not exist; that is, $h$ is not proportional to $t^{\frac{1}{2}}$. We define $\theta_{L}$, the value of $\theta$ at $x=L$, by

$$
L=h \int_{\Theta_{L}}^{1} f D d \theta
$$

and the amount of material within the layer, which is equal to $S t^{\frac{1}{2}}$ for $t \leq t_{T}$ is now defined by

$$
M=\Theta_{L} L+h \int_{\Theta_{L}}^{1} \theta f D d \Theta
$$

or integrating by parts,

$$
M=L-h \int_{\Theta_{L}}^{1}(1-\theta) f D d \theta
$$

The presence of $(1-\theta)$ means that the last term in (18) is of order $O[E L]$. Changes in the definition of $f$ introduces relative errors $O(\varepsilon)$. Hence any choice of $f$ will affect $M$ by a term of order $O\left(\varepsilon^{2} L\right)$, which means that the relative error $O\left(\varepsilon^{2}\right)$ remains of the order of the error for $t<t_{T}$. By comparison $\theta_{L}$, defined by [16], is not optimal and has an error $\Theta(\varepsilon)$.

The value of $h$ must be chosen so that $M=S t_{T}^{\frac{1}{2}}$ at $t=t_{T}$. This is easily obtained remembering that (12), which yields $S$, results from integrating ( 7 ) twice. Then, integrating (1) twice, we obtain, 


$$
\int_{\Theta_{L}}^{1} D d \theta=\int_{0}^{L} d x \int_{x}^{L}(\partial \theta / \partial t) d x
$$

or, from (15),

$$
\int_{\Theta_{L}}^{1} D d \theta=h h \int_{\Theta_{L}}^{1} d \Theta\left[\int_{\Theta}^{1} f D d \bar{\theta}\right]^{2} .
$$

This equation gives $h(t)$ for any convenient choice of $f$ to obtain $M$ with an error $o\left(\varepsilon^{2}\right)$.

\section{Application}

To apply the foregoing results some explicit $D(\theta)$ relation must be chosen. Because of its importance in practice we shall use as an example the relation given in (2). Then (14) and (13) yield

(21) $D_{0} t_{T} / L^{2}=n e^{-n}\left[\left(1-n^{-1}\right)\left(1-e^{-n}\right)+e^{-n}\right]^{2} /\left\{\left(1-e^{-n}\right)^{2}\left[\left(1-e^{-n}\right)\left(2-n^{-1}\right)+e^{-n}\right]\right\}$, with

$$
M=\left(t / t_{T}\right)^{\frac{1}{2}} L\left[\left(1-e^{-n}\right)^{-1}-n^{-1}\right]
$$

for $t \leq t_{T}$. Note that as soon as $n$ is sufficiently larger than one, which is necessary for $\varepsilon$ to be small, then $e^{-n}$ is negligible compared to one in (21) and (22). For $t>t_{T},(20)$ is easily integrated taking $f=1$, for instance, to yield

$$
M / L=1+n^{-1}\left[\ln u-(1-u)^{-1} \ln u-1\right],
$$

where $u$ stands for

$$
u=\exp \left[n\left(\Theta_{L}-1\right)\right]
$$

and is related to time by

$$
\begin{aligned}
& 3 D_{0} L^{-2}\left(t-t_{T}\right) e^{n}=-(1-u)^{-3} \ln u-n\left(1-e^{-n}\right)^{-3}+ \ln u \\
&+n-\ln \left[(1-u) /\left(1-e^{-n}\right)\right]-\frac{1}{2}(1-u)^{-1}+\frac{1}{2}\left(1-e^{-n}\right)^{-1} \\
&-(1-u)^{-2}+\left(1-e^{-n}\right)^{-2}
\end{aligned}
$$


To apply (23) and (25) a value of $u$ is chosen between $e^{-n}$ and .I ; then $t>t_{T}$ is obtained from (25) and the corresponding value of $M$ is given by (23) .

In order to estimate the accuracy of (23) and (25) it is necessary to obtain a numerical solution of (1), the details of which have been presented elsewhere, [3]. It is only necessary to point out here that the numerical scheme is more useful when $n$ is small, as increasing $n^{\prime}$ s require rapidly inordinate amounts of computer time and are too expensive. In practice we could not apply the numerical scheme for $n>4$. However, the case $n=4$ is specially interesting as it should provide a lower limit for the application of the optimal results. That is, since errors on $M$ are of order $O\left(\varepsilon^{2}\right)$ or $O\left(n^{-2}\right),(23)$ and (25) should have at most a few per cent error for $n=4$; the error becoming smaller for increasing $n^{\prime} s$.

Figure 1 gives $M / L$ as a function of $\left(t D_{0} / L^{2}\right)^{\frac{1}{2}}$ with $t_{T} D_{0} / L^{2} \simeq 0.025$ for $n=4$ given here in (23) and (25). The maximum error is about $3 \%$ for $t D_{0} / L^{2} \simeq 0.04$ which is of the order of $n^{-2} \simeq 6 \%$. as expected. By comparison $\Theta_{L}\left(D_{0} t / L^{2}\right)$ or $\Theta(x / L)$ for a given time and calculated with $f=1$ are given in Figures 2 and 3 and show a much larger error, specially for $t \simeq t_{T} \cdot \theta_{L}$ is obtained from (24) and (25) and $\theta(x / L)$ is given by, from (15) and (16),

$$
x / L=\left(e^{n}-e^{n \Theta}\right) /\left(e^{n}-e^{n \Theta} L\right)
$$

As expected the error for $\theta$ shown in Figures 2 and 3 is large. However Figure 3 shows that on the average, $M=\int \theta d x$, is obviously accurate as already observed in Figure 1. This, of course, results from the fact that $M$ is optimal even though $\theta$ is not.

It is interesting that the optimal result for $M / L$ remains accurate even for $n=1$ as shown in Figure 4. The maximum error is only of the order of $5 \%$. This is surprising since the method was developed on the 


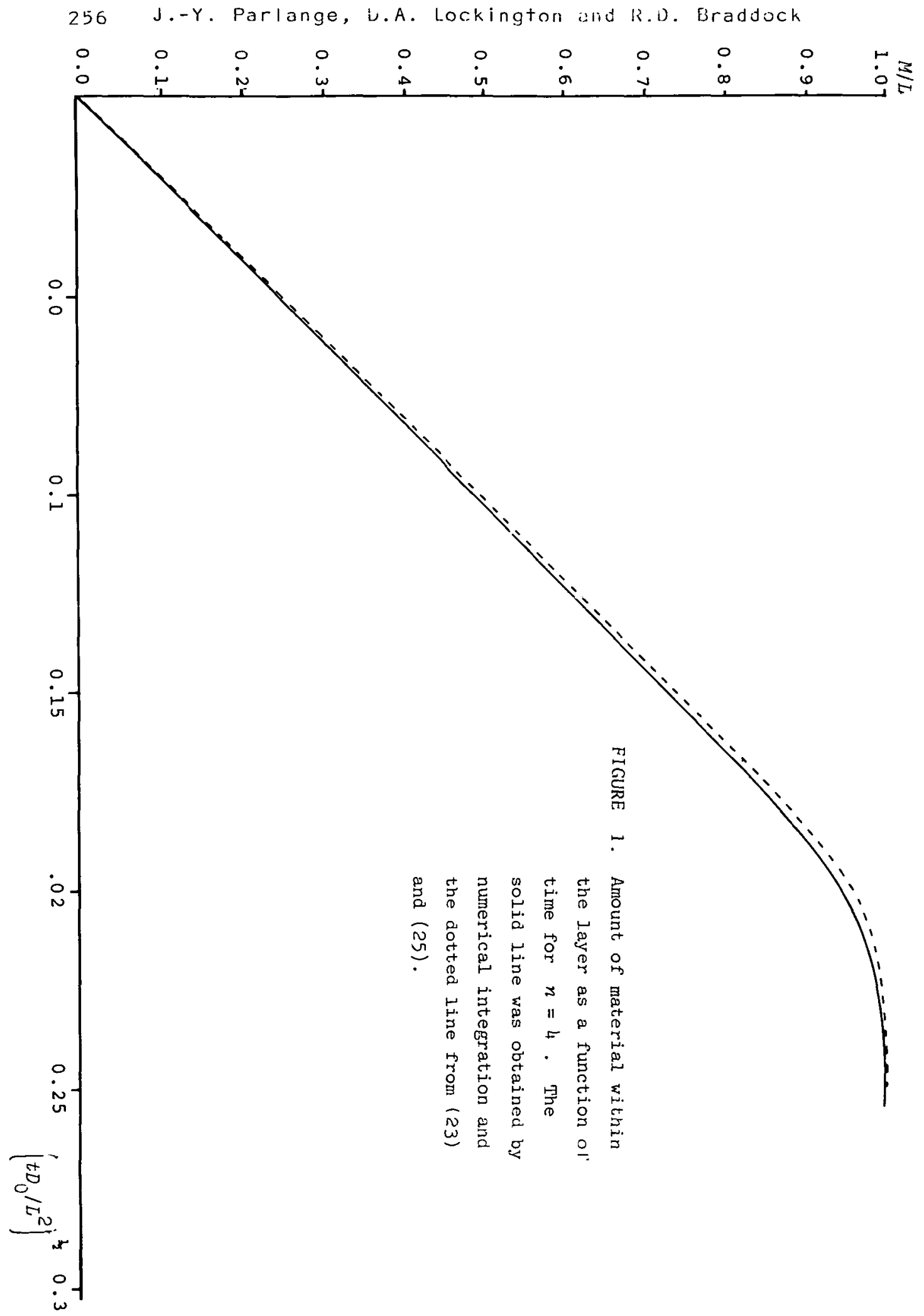




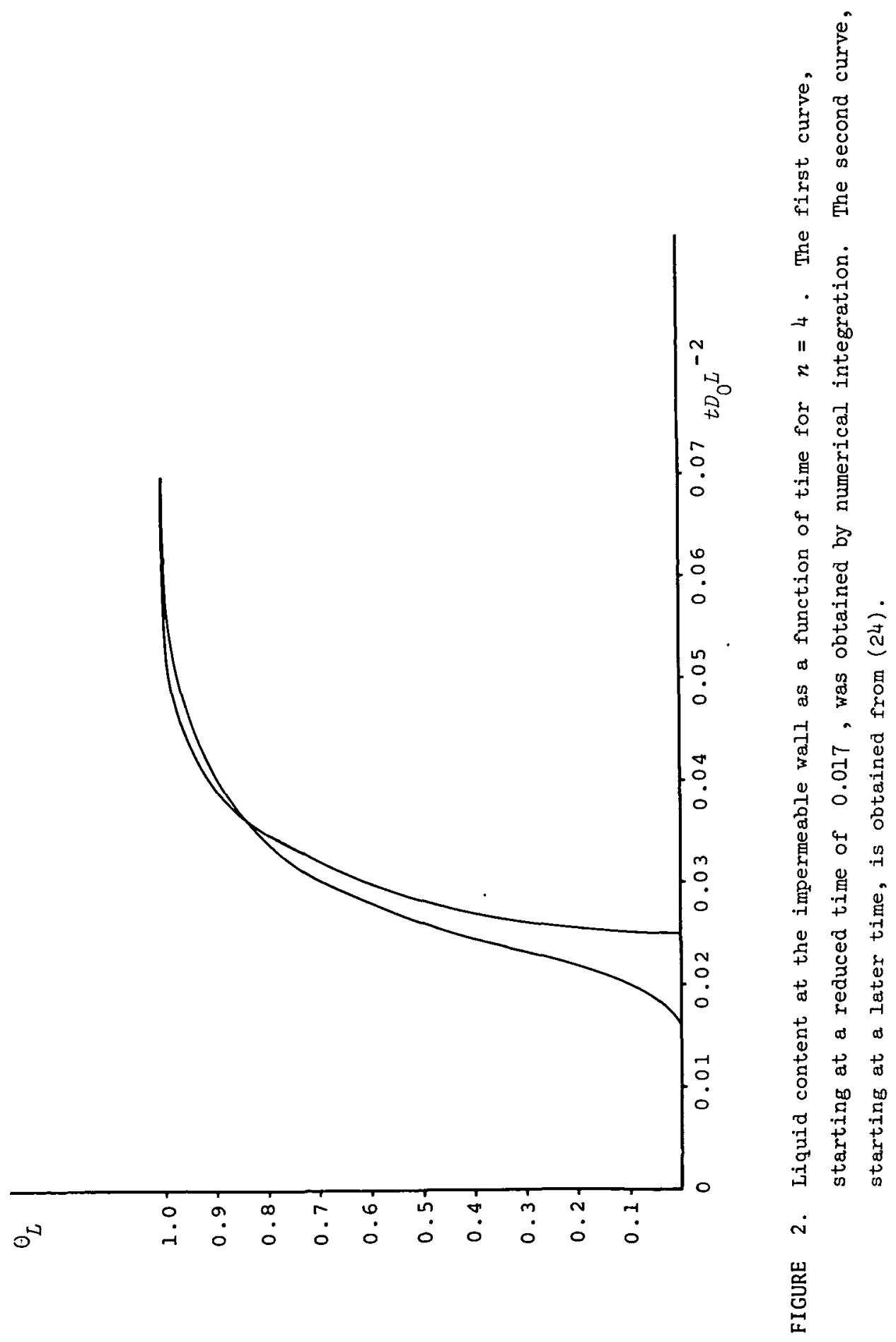


蛋

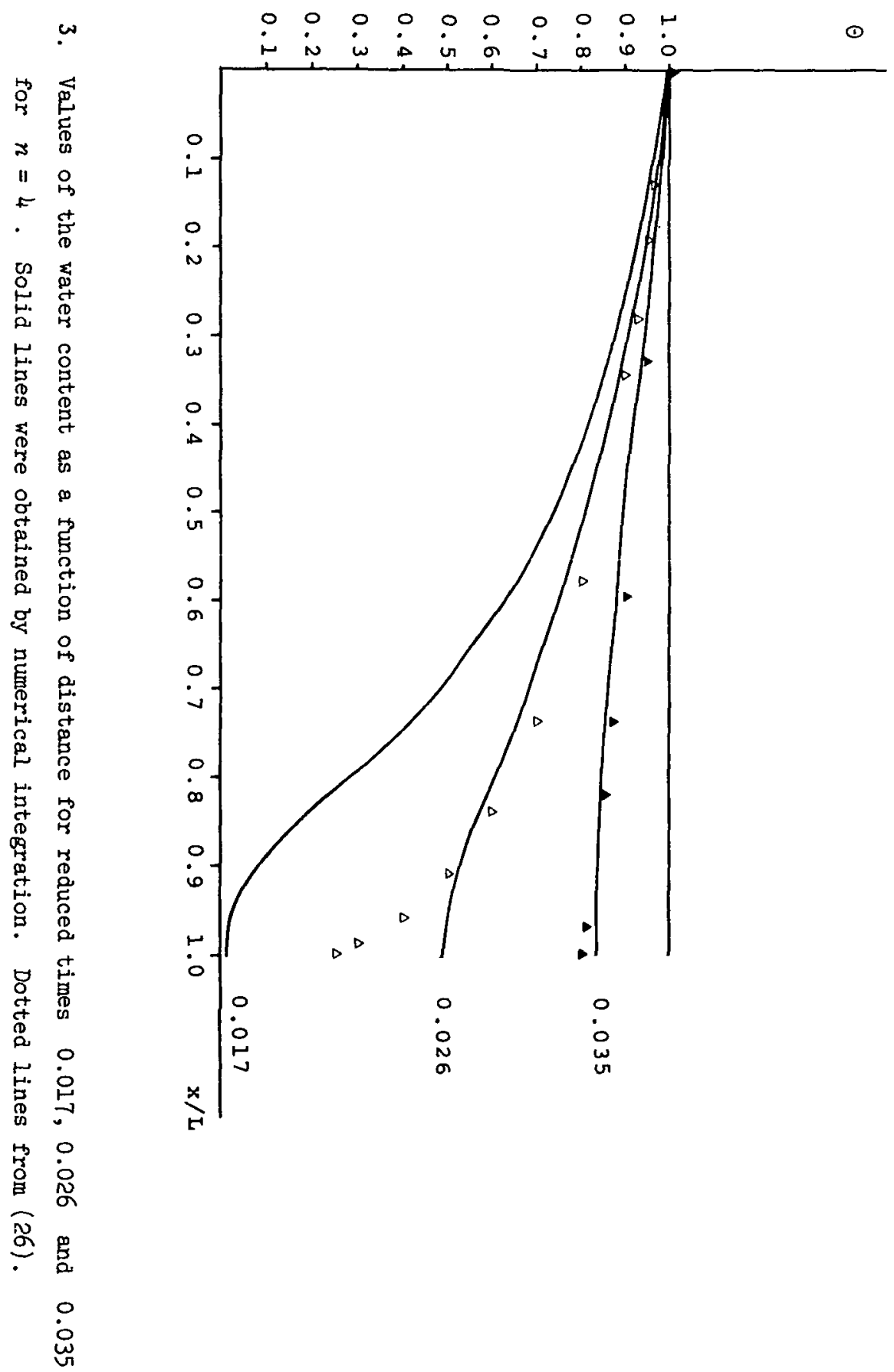




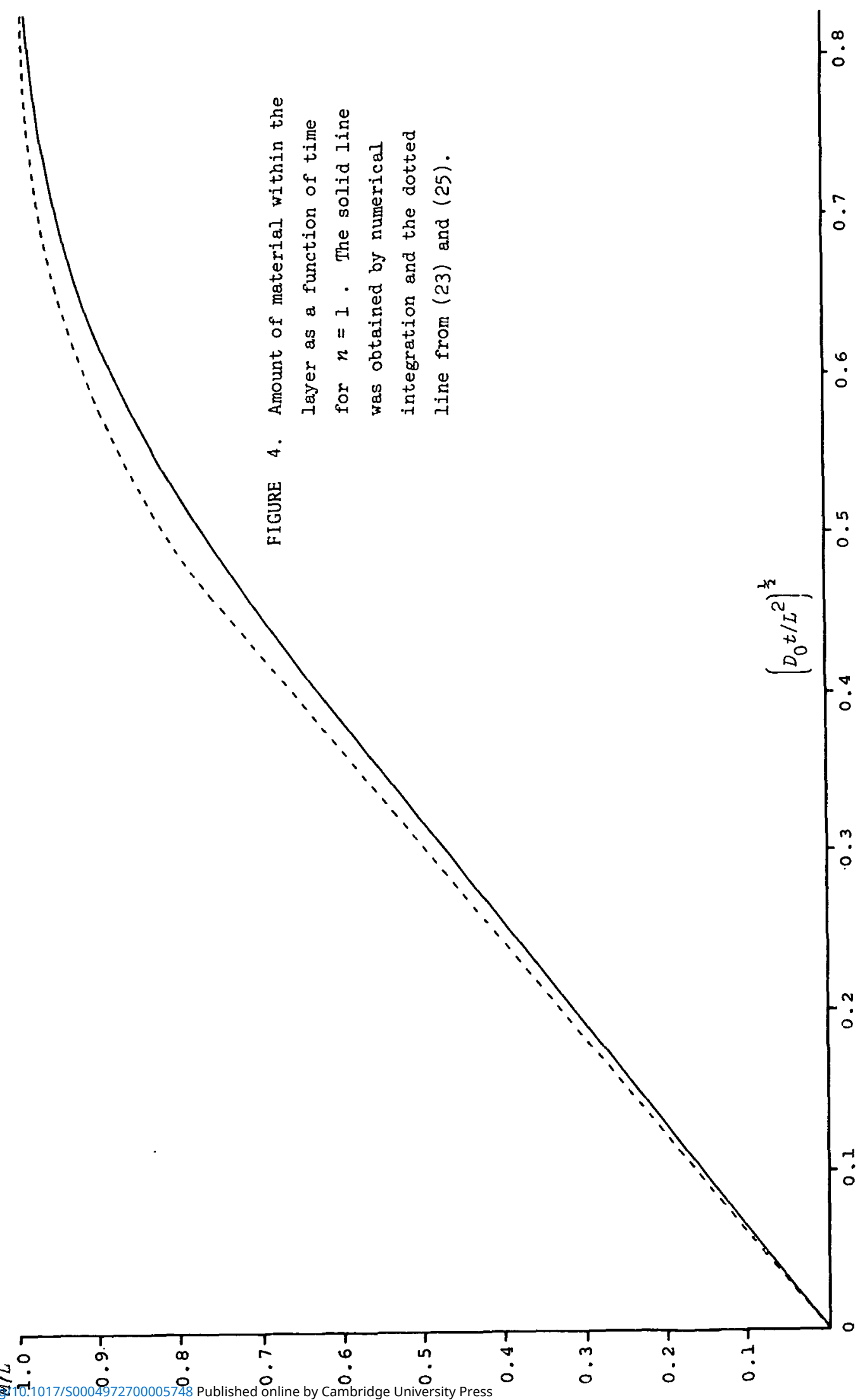


basis of $n$ large. Such agreement was observed earlier for sorptivity as well, [4], [13]. It is not clear why the prediction should be so good for $n$ small.

In conclusion, the excellent agreement for $n=4$ points out that for $n>4$, for example $n=8$ for a soil, [19], the optimal formulation can be used with confidence, while no practical numerical procedure is presently available. Finally if a better profile $\Theta(x, t)$ was desired, an iterative procedure is available, [11], where $\partial \Theta / \partial t$ in (1) is estimated from (15) and a new $\Theta$ is calculated.

\section{References}

[1] N. Anderson and A.M. Arthurs, "Dual extremum principles for a nonlinear diffusion problem", Quart. Appl. Math. 35 (1977/8), $188-190$.

[2] R.D. Braddock and J.-Y. Parlange, "Some accurate numericaI solutions of the soil-water diffusion equation", Soil Sci. Soc. Amer. J. $44(1980), 656-658$.

[3] R.D. Braddock, J.-Y. Parlange, D. Lockington and P. Doilibi, "Nonlinear diffusion with a barrier", Numerical solutions of partial differential equations (North-Holland, Amsterdam, to appear).

[4] R.O. Braddock, J.Y. Parlange, and I.G. Lisle, "Properties of the sorptivity for exponential diffusivity and application to the measurement of the soil water diffusivity", Soil Sci. Soc. Amer. J. $45(1981), 705-709$.

[5] R.R. Bruce and A. Klute, "The measurement of soil moisture diffusivity", Soiz Sci. Soc. Amer. J. 20 (1956), 458-462.

[6] W. Brutsaert, "The concise formulation of diffusive sorption of water in a dry soil", Water Resour. Res. 10 (1974), 1118-1124.

[7] C. Matano, "On the relation between the diffusion coefficients and concentration of solid materials (the nickel-copper system)", Japan. J. Phys. 8 (1933), 109-113. 
[8] Jean-Yves Parlange, "On solving the flow equation in unsaturated soils by optimization: horizontal infiltration", Soil Sci. Soc. Amer. J. 39 (1975), 415-418.

[9] J.-Y. Parlange, "Conment", Water Resour. Res. 11 (1975), 1040-1041.

[10] J.-Y. Parlange, "Water transport in soils", Ann. Rev. Fluid Mech. 12 (1980), 77-102.

[11] J.-Y. Parlange and R.D. Braddock, "An application of Brutsaert's and optimization techniques to the nonlinear diffusion equation: the influence of tailing", Soil Sci. 129 (1980), 145-149.

[12] J.-Y. Parlange and R.D. Braddock, "A note on some similarity solutions of the diffusion equation", 2. Angew. Math. Phys. 31 (1980), 653-656.

[13] J.-Y. Parlange, R.D. Braddock, and B.T. Chu, "First integrals of the diffusion equation: an extension of the Fujita solutions", Soil Sci. Soc. Amer. J. 44 (1980), 908-911.

[14] J.-Y. Parlange, R.D. Braddock, and I. Lisle, "Third-order integral relation between sorptivity and soil water diffusivity using Brutsaert's technique", Soil Sci. Soc. Amer. J. 44 (1980), $889-891$.

[15] J.-Y. Parlange, R.D. Braddock, and G. Voss, "Two-dimensional similarity solutions of the nonlinear diffusion equation from optimization and first integrals", Soil Sci. 131 (1981), 1-8.

[16] J.-Y. Parlange, R.D. Braddock, G. Sander and F. Stagnitti, "Three dimensional similarity solutions of the nonlinear diffusion equation from optimization and first integrals", J. Austral. Math. Soc. Ser. B 23 (1982), 297-309.

[17] G.J. Pert, "A class of similar solutions of the non-linear diffusion equation", J. Phys. Ser. A: Math. General 10 (1977), 583-593.

[18] J.R. Philip, "Flow in porous media", Ann. Rev. Fluid Mech. 2 (1970), 177-204. 
262 J.-Y. Parlange, D.A. Lockington and R.D. Braddock

[19] K. Reichardt, D.R. Nielsen, and J.W. Bìggar, "Scaling of horizontal infiltration into homogeneous soils", Soil Sci. Soc. Amer. J. 36 $(1972), 241-245$.

School of Australian Environmental Studies,

Griffith University,

Brisbane,

Queensland 4111 ,

Australia. 